\title{
EFFORTS ON GENDER BALANCE CAPACITY BUILDING IN GIT
}

\author{
P. Tripathi *, R. B. Thapa \\ International Centre for Integrated Mountain Development (ICIMOD), Kathmandu, Nepal- Poonam.Tripathi@icimod.org
}

Commission V, WG V/7 \& Commission IV, WG IV/6

KEY WORDS: Capacity building, GIT, HKH, Gender

\begin{abstract}
:
The Hindu Kush Himalaya (HKH) region is among the most discrete and diverse region facing various ecological, environmental and socio-economic threats in terms of increasing demands for natural resources and its consequences in the form of overexploitation, disaster, droughts, extreme weather, and climate change etc. Geospatial information technology (GIT) with Earth observation (EO) data are effectively supporting the implementation of development agendas in HKH by providing extensive solutions to above-pressing issues by not only addressing them but also providing services in daily life. These technologies have effectively bolstered in time via innovation, creating jobs and confidence in people that supports filling the data and knowledge gaps in the region. However, the involvement and participation of women in GIT is mere in the region despite their vital role in environmental management and decision making. Realizing the issue, we acknowledged and implemented the twin challenges i.e. capacity building and gender equality for building the pathways to sustainable development via innovative steps and processes to bridge the gender imbalance in GIT workforce in HKH. For the purpose, we organized various capacity building trainings and workshops with a broad focus towards GIT applications in forest, agriculture, water management, drought and climate change along with the hands-on exercises. In addition, specific women focused training programs i.e. Empowering Nepali Women through Technology Training and Women in GIT were organized during 2017 and 2018 respectively. These efforts delivered optimistic results in terms of building confidence, decision making and more women participation showing an increment of $\sim 5 \%$ participation by women in 2017-2018 fiscal year with respect to 2016-2017 fiscal year. In HKH nations with less social parity, the information delivered by this gender mainstreaming effort will have life-changing implications to achieve workforce parity.
\end{abstract}

\section{INTRODUCTION}

As one of the most diverse mountains, the Hindu Kush Himalaya (HKH) region serves to nearly 240 million population through various ecosystem services i.e. food, timber, fiber, fresh air and water, climate regulation, carbon storage, soil protection and the maintenance of aesthetic, cultural, and spiritual values (Hamilton 2015; Wester et al., 2019). However, the region faces multiple challenges in terms of various ecological, environmental and sociocultural/economic securities due to the increasing demands of the natural resources (Wester et al., 2019). The unsustainable activities like overexploitation, rapid urbanization, land use land cover (LULC) changes and, habitat fragmentation has led to a profound impact on the sustainability and resilience of the region along with the threat to sustain the large population of the HKH region (Thapa et al., 2019; Wester et al., 2019).

Geospatial information technology (GIT) and earth observation (EO) data has played a vital role in addressing and mitigating the above pressing issues and have effectively bolstered via innovative technology, creating jobs and confidence in people. The application of GIT as a decision-making tool via data and information management, spatial data collection, quality assessment, processing and, modelling have revealed many new insights to knowledge gaps in ecology-environment interactions, agricultural analysis and natural disaster etc.
(Ingole et al. 2015; Manfré et al. 2012; Ofori-Amoah, 2008). However, the effectiveness of GIT depends on the people who know the theory and applications of this technology, which is lacking in the developing countries (Merino and Carmenado 2012; World Bank 2005). Capacity building is the core strength of any sustainable development via enhancing and accelerating individual, institutional and infrastructure capacity and assisting to the use of advanced technology and innovations for decision making that provides options in bridging the science and policy gaps. In addition, despite the vital role of women in environmental management and decision making their involvement and participation in GIT is very low in Asia particularly in HKH region (Sherpa 2007; Mehta, 2007) that might lead to a large gender imbalance around the globe (Caitlin 2014). To overcome these challenges, a robust approach of capacity building $(\mathrm{CB})$ is required that enhances the technical abilities of society and countries at individual, organizational and institutional levels to implement and achieve the development goals (Thapa et al., 2019; Chandler and Kennedy 2015). Recently, Thapa et al. (2019) developed a sequential work flow for capacity building highlighting four major tasks as Assessment, Design, Implementation, and Monitoring (ADIM).

SERVIR-HKH program, a joint development initiative of USAID and NASA, works on bridging these gaps in the HKH region (http://servir.icimod.org). The program supports $\mathrm{CB}$

\footnotetext{
* Corresponding author
} 
activities for government institutions and other development stakeholders to incorporate GIT and EO data into decision making process. Wherein, the knowledge and information is delivered through national and regional platforms and collaborations through development of innovative, user-tailored analyses, decision-support products, and distinct trainings (SERVIR Global 2018). This paper addresses the gender balanced approach for the CB of HKH region.

\section{MATERIALS AND METHODS}

\subsection{Capacity building strategy:}

Our study area, the HKH region includes 8 member countries namely Afghanistan, Bangladesh, Bhutan, China, India, Myanmar, Nepal and, Pakistan (Figure 1). We followed the sequential CB workflow given by Thapa et al. (2019) that addresses four major tasks as assessment, Design, Implementation, and Monitoring (ADIM). Wherein, the capacity of the targeted institutions were assessed to identify gaps followed by design of $\mathrm{CB}$ workflow incorporating the selection of topic and subject matter experts (SMEs), curriculum and materials development, delivery methods, and execution plan. Further the implementation of the CB activities were carried out via various training programs during the years 2015 to 2019 in which gender balance among the participants are given high priority. The concern to bridge the gender gap urged us to introduce the gender strategy to mainstream gender and equity issues into GIT to provide equal opportunities to male and female in gaining and sharing knowledge and experience and to disseminate it via accessible opportunities. These issues are prominently addressed in the Medium-Term Action Plan 2018-2022 - ICIMOD (MTAP IV). For the purpose, we keep motivated our partners to respect gender equity and encourage providing equal opportunity of participation for all genders during training invitation and nomination process. We delivered the trainings in four categories (table1) as: 1) exposure and learnings (EL), 2) standard trainings (ST), 3) on the job training (OJT), 4) training of the trainers (TOT), with a broad focus towards GIT applications in forest, agriculture, water management, drought and climate change along with the hands-on exercises.

Special focus is needed to attract potential female students and professionals to adopt career in GIT field. Therefore, we offered special trainings designed for women participants exclusively to develop gender balance workforce in the HKH region. For the purpose, women oriented trainings i.e. Empowering Women in Geospatial Information Technology (GIT) were also organized. The evaluation of the trainings were done by a pre and post training survey based on participant's self-knowledge on the subject and technical skill to understand the knowledge gain in different topics.

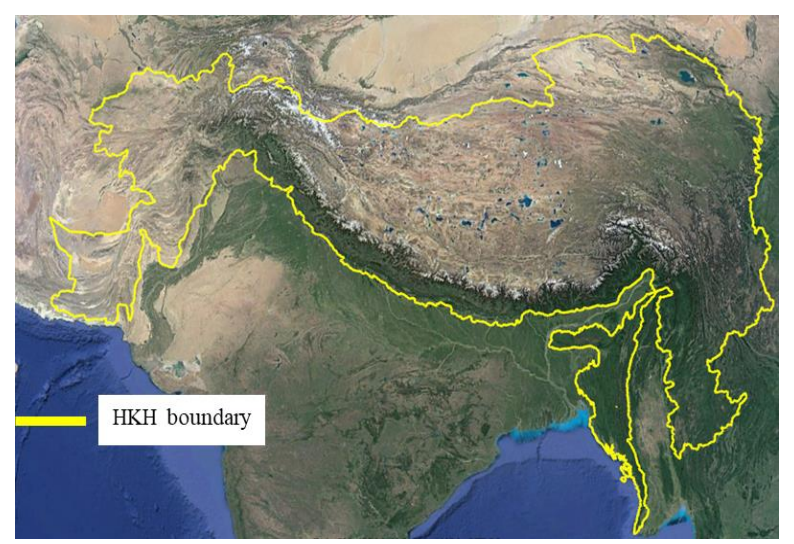

Figure 1. The Hindu Kush Himalaya region

\section{RESULTS AND DISCUSSION}

\subsection{Capacity building events}

We organized different capacity building trainings in the region during October 2015 to September 2019. Overall, 62 trainings were organized under four major type as EL, ST, OJT and TOT as mentioned in methods section. Wherein, 1, 19, 19 and, 23 training events were organized in the fiscal year 2015, 2016, 2017, and 2018 respectively. As a whole, approx.1360 participants participated in these events including $~ 67 \%$ of participation by male and $\sim 33 \%$ of the participation by females respectively (Figure 2).

\subsubsection{Year wise capacity building analysis}

Year wise statistics showed overall $\sim 67 \%, \sim 62 \%, \sim 74 \%$ participation of male candidates for the fiscal year 2016, 2017 and 2018 respectively. On the other hand, female participation was observed as $\sim 33 \%, \sim 38 \%$ and, $\sim 25 \%$ respectively for the fiscal years 2016, 2017 and 2018. A significant gain of $\sim 5 \%$ in the participation of women during the fiscal year 2017-2018 compared to the year 2016-2017 was observed. This highlighted the successful approach of implementing gender strategy into the action plan of ICIMOD. We observed an overall drop of $\sim 10 \%$ in the women participation during the fiscal year 2018-2019. This might be attributed to the irrelevant nomination procedure of the collaborating partners (Thapa et al., 2019). In addition, engagement of fewer women in GIT and EO based professions in the HKH region might have led to their lower participation.

\section{Participation in percentage}

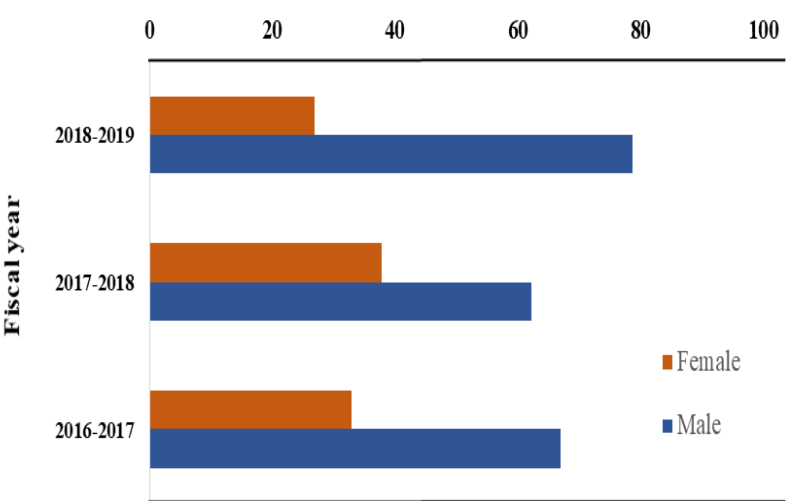

Figure 2. Year-wise comparison of the male and female participation (\%) in capacity building events 


\subsubsection{Service area based capacity building analysis}

The broad topics chosen during the training covered applications of GIT and EO in the forest, agriculture, water management, drought and climate change. Wherein, the basic theory and hands-on exercises were designed for the participants to understand and implement the knowledge. These broad topics were categorized into five major categories as Agriculture and Food Security (AFS), Water Resources and Hydro-climatic Disasters (WRHD), Land Use Land Cover and Ecosystem (LULC\&E), Weather and Climate Services (WCS) and, cross cutting (CC; Figure 3). Wherein, 8, 21, 13, 6 and 14 events were organized under AFS, CC, LULC\&E, WCS and WRHD respectively (Figure 3 ). In these events maximum participation from female were observed in CC area (approx.48\%) however, the least participation was observed under the service theme WCS (approx.17\%).

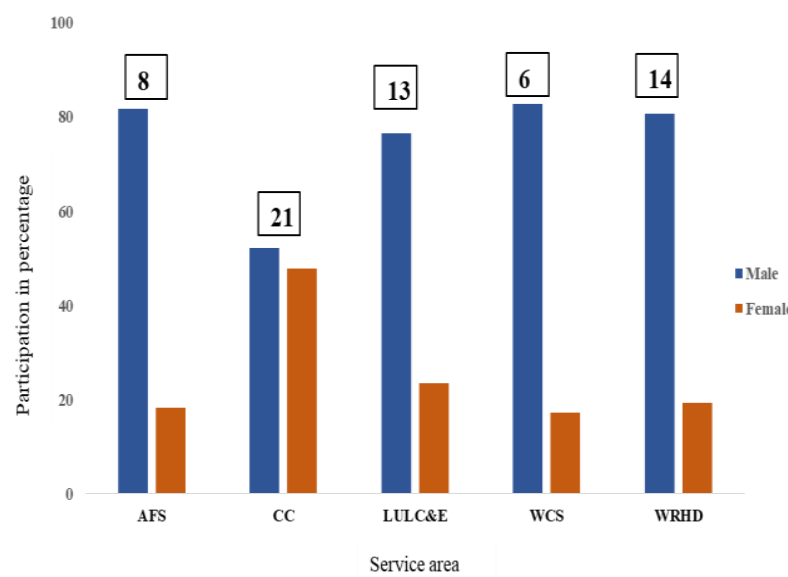

Figure 3. Service area wise comparison of the male and female participation (\%) in capacity building events

* In boxes overall number of capacity building events during the years 2015 to 2019

**AFS: Agriculture and Food Security; CC: Cross cutting;

LULC\&E: Land Use Land Cover and Ecosystem; WCS:

Weather and Climate Services; WRHD: Water Resources and Hydro-climatic Disasters

\subsubsection{Training type based capacity building analysis}

Based on the four types of CB trainings (i.e. EL, OJT, TOT and, ST) we observed overall $8,5,5$ and 44 events were organized during the years 2015 to 2019. In these maximum participants were observed in the standard trainings. We observed the least participation of women in the OJT trainings showing only $\sim 9 \%$ and maximum participation in the EL trainings with $\sim 41 \%$. In contrast, male participation was observed to be maximum for OJT trainings $(\sim 90 \%)$ and least participation for EL trainings ( 58\%) respectively (Figure 4). This statistics gives an insight of the differences in opportunities for male vs female participants that shows male participants are more into GIT and EO professions compared to female participants. While young women are looking for the opportunities to build their confidence and GIT as a career option via EL.

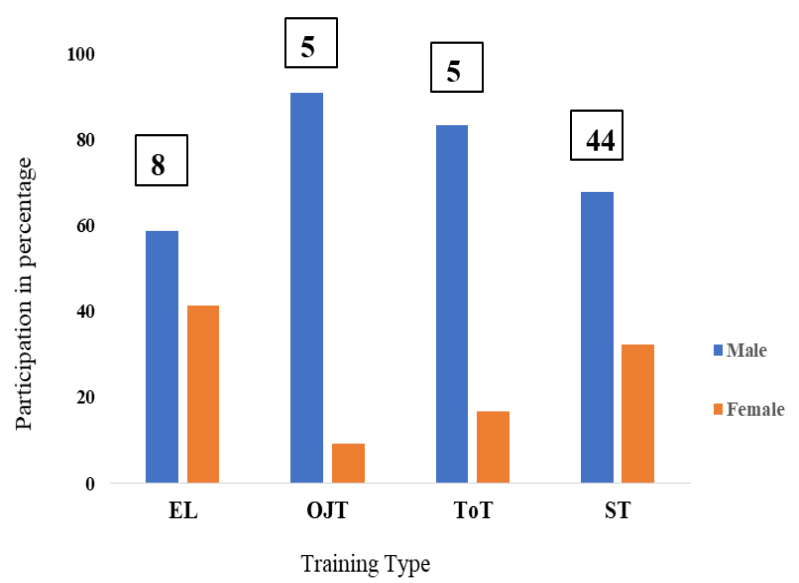

Figure 4. Training type wise comparison of the male and female participation (\%) in capacity building events

* In boxes overall number of capacity building events during the years 2015 to 2019

** EL: exposure and learnings; OJT: on the job training; TOT: training of the trainers; ST: standard trainings

\subsection{Women focused program:}

Realizing the need to involve females in various thematic programs to opt for their career in GIT field we organized and supported various women oriented programs. For the purpose, training on "Empowering Women in Geospatial Information Technology (GIT)" was organized in 2018 and 2019 respectively. Overall, 84 and 40 women participants attended the training during 2018 and 2019 respectively and were benefitted. This training focused for women attained a massive attention from media under the headline "Empowering Nepali Women through Technology Training" and was a successful event in 2018.

As one of the innovative ways to promote women in science and technology we supported Miss Tech 2017, a massive national collaboration, focused on promoting women technopreneurship that takes place to solve the issue in the society faced by Women with the theme Mountain Women "Transformational Changes through Technology". In this programme, 52 participants including 45 amateurs and 7 professionals collaborated, helping each other into inspiring creative and innovative solutions to the challenges. A total of 16 teams participated and they worked on solving challenges faced by women living in the mountains. This event was duly acknowledged in the media as "Women drive innovation at Miss Tech, 2017" under the news cover of "The Himalaya".

These trainings not only provided more opportunities to the professionals working in GIT and EO field but also enhanced the confidence in the participants to opt this field for their career and is well evidenced.

\subsection{Monitoring and evaluation}

As an integral part of $\mathrm{CB}$ monitoring and evaluation serves the effective role in assessing the actual capacity build via trainings. Therefore, we conducted pre-and-post assessments for each type of capacity building activity. The pre assessment provides guidelines about the participant's awareness and expectations on the capacity building content. Wherein, we provided the questions on the participant's basic awareness of the subjects through self-assessment and then a technical 
session to assess their skill level. The post assessment provided the level of knowledge acquisition by the participants and feedback for the continuous improvement.

\section{CONCLUSION}

Although, innovations via GIT has led to a growing number of projects in various sectors to address and improve ecosystem sustainability, community infrastructure, public health etc. in the HKH region; the lack of human resources and capacity from individual, institutional and national level hinders the implementation in effective ways. Therefore, we presented a robust and simple approach ADIM designed by Thapa et al., (2019) to build the capacity of the region.

We conducted overall 62 capacity building programmes in the $\mathrm{HKH}$ region successfully via ADIM's approach which clearly indicated its effectiveness in individual, institutional $\mathrm{CB}$ and in improving the gender gap in the GIT field of the HKH region. The specific programs for women identified the expectations and an opportunity for career options in GIT for the women participants. However, there is still much scope to improve the activities in future. We believe, that the information delivered by this gender mainstreaming effort will have life-changing implications to achieve workforce parity in the HKH nations with balanced gender involvement.

\section{REFERENCES}

Caitlin Dempsey (2014). Gender in the GIS Workforce. Article published on June 2014 in GIS career and jobs https://www.gislounge.com/gender-gis-workforce/).

Hamilton, L. S., 2015. When the sacred encounters economic development in mountains. George Wright Forum 32(2), 132 140 .

Ingole, N. A., Ram, R. N., Ranjan, R., \& Shankhwar, A. K., 2015. Advance application of geospatial technology for fisheries perspective in Tarai region of Himalayan state of Uttarakhand. Sustainable Water Resources Management. 1(2), pp.181-187.

Manfré, L.A., Hirata, E., Silva, J.B., Shinohara, E.J., Giannotti, M.A., Larocca, A.P.C. and Quintanilha, J.A., 2012. An analysis of geospatial technologies for risk and natural disaster management. ISPRS International Journal of GeoInformation, 1(2), pp.166-185. https://doi. org/10.3390/ijgi1020166.

Mehta. M., ICIMOD, 2007, Draft Gender and Disasters Report.

MTAP-IV Medium-Term Action Plan 2018-2022 - ICIMOD.

Ofori-Amoah, B., 2008. Building Capacity to Use Geospatial Technology for Development in Africa: Lessons from the Uganda GIS Project. Global Dialogue on Emerging Science and Technology (GDEST) Cape Town, South Africa, pp.20012009.

SERVIR Global, 2018. A Retrospective Report: 2014-2018. www.servirglobal.net
Sherpa, D., 2007. New vulnerabilities for mountain women: A different light on the Greater Himalaya. Status Report, International Centre for Integrated Mountain Development (ICIMOD), Kathmandu, Nepal.

Thapa, R.B., Matin, M.A. and Bajracharya, B., 2019. Capacity Building Approach and Application: Utilization of Earth Observation Data and Geospatial Information Technology in the Hindu Kush Himalaya. Frontiers in Environmental Science.

Wester P., Mishra A., Mukherji A., Shrestha A. B., 2019. The Hindu Kush Himalaya Assessment-Mountains, Climate Change, Sustainability and People, Springer Nature Switzerland AG, Cham.

World Bank (2005), Capacity building in Africa - an OED evaluation of World Bank support. Operation Evaluation Department, Washington, D.C. (No. 34351). 